\title{
EFECTO DE LA SUSTANCIA P SOBRE LA PRESIÓN ARTERIAL PULMONAR EN POLLOS EXPUESTOS A LA ALTURA
}

\author{
Sebastiana Bernilla De la C. ${ }^{1}$, Sergio Cueva M. ${ }^{2}$ y Milder Ayón S. ${ }^{2}$
}

\section{Abstract}

Hypoxia at high altitude produces pulmonary arterial hypertension as a result of pulmonary arteriolar vasoconstriction, but the mechanism of action is still unknown. Under these conditions, vasoactive substances like Substance P (SP), are synthesized in the vascular endothelium. To study the effect of SP on the mean pulmonary arterial pressure (PAPm), 30 male chickens for meat production, born and reared at sea level were used. The birds were exposed at 35 days of age to 3,320 $\mathrm{m}$ of altitude for 3 days, and then the PAPm was determined by catheterising the pulmonary artery, both before (Pre-SP) and after (Post-SP) injection of SP in doses of $0.30 \mathrm{mcg}(0.3 \mathrm{ml}) / \mathrm{kg}$ of body weight. The mean value of PAPm for Pre-SP was $37.92 \pm 4.10 \mathrm{~mm} \mathrm{Hg}$, while for Post-SP at the times of $01,05,10,15,30,45,60,75$ and 90 seconds were $36.08 \pm 3.70,31.11 \pm 7.51,28.00 \pm 10.22$, $26.43 \pm 11.02,24.43 \pm 11.02,23.17 \pm 11.54,24.08 \pm 10.98,27.27 \pm 10.97$, and $30.67 \pm 9.40 \mathrm{~mm}$ $\mathrm{Hg}$, respectively. SP significatively diminished the PAPm $(\mathrm{p}<0.001)$ from the 1 st second and reached his lowest value at 45 seconds. The quick decrease of PAPm suggests a possible influence of SP at vascular endothelial level through its vasodilator effect, decreasing the press effect that could be related to the hyperpolarization of the membrane as well as to an increase of nitric oxide activity.

Key words: Substance P, hypoxia, chicken, pulmonary arterial pressure, tachykinin

\section{Resumen}

La hipoxia de la altura produce hipertensión arterial pulmonar como consecuencia de una vasoconstricción arteriolar pulmonar, cuyo mecanismo es aún desconocido. Bajo estas condiciones, en el endotelio vascular se sintetizan sustancias vasoactivas en las que podría incluirse la Sustancia P (SP). A fin de determinar el efecto de la SP sobre la Presión Arterial Pulmonar media (PAPm) se utilizaron 30 pollos de carne, machos, nacidos y criados a nivel del mar. A los 35 días de edad fueron expuestos a 3,320 m de altitud por 3 días, al término de los cuales se determinó la PAPm por cateterismo directo de la arteria pulmonar, tanto antes (Pre-SP) como despues de la administración de Sustancia P (Post$\mathrm{SP})$, en dosis de $0.30 \mathrm{mcg}(0.3 \mathrm{ml}) / \mathrm{kg}$ de peso vivo. El valor promedio de la PAPm para PreSP fue de $37.92 \pm 4.10 \mathrm{mmHg}$, mientras que en los tiempos de 01, 05, 10, 15, 30, 45, 60, 75 y 90 segundos para Post-SP fueron $36.08 \pm 3.70,31.11 \pm 7.51,28.00 \pm 10.22,26.43 \pm 11.02$, $24.43 \pm 11.02,23.17 \pm 11.54,24.08 \pm 10.98,27.27 \pm 10.97$ y $30.67 \pm 9.40 \mathrm{~mm} \mathrm{Hg}$, respectivamente. La SP disminuyó significativamente la PAPm desde el primer segundo ( $<<0.001)$, y alcanzó su mínimo valor a los 45 segundos. La rápida disminución de la PAPm permite

\footnotetext{
${ }^{1}$ Graduada de la Unidad de Postgrado, FMV-UNMSM. Práctica privada

${ }^{2}$ Laboratorio de Fisiología Animal, FMV-UNMSM
} 
sugerir la posible influencia de la SP a nivel endotelial vascular por su acción vasodilatadora, disminuyendo el efecto presor que, a su vez, podría estar relacionado con la hiperpolarización de la membrana o con el aumento de la actividad del óxido nítrico.

Palabras clave: Sustancia P, hipoxia, pollos, presión arterial pulmonar, taquicininas

\section{INTRODUCCIÒN}

La baja presión de oxígeno en la altura produce una vasoconstricción arteriolar pulmonar, principal mecanismo causante de la hipertensión arterial pulmonar (HAP) conducente a una insuficiencia cardiaca derecha que frecuentemente culmina con la muerte del animal. Este cuadro patogénico se presenta en diversas especies animales, especialmente en bovinos (Alexander et al., 1960; Grover, 1966; Will y Bisgard, 1975) y en aves (Burton et al., 1968; Cueva et al., 1974), ocasionando grandes pérdidas económicas.

Se han realizado diversos estudios sobre el tema, pero aun no se logra encontrar el mecanismo regulador íntimo de esta condición. Actualmente se conoce que existen factores intrínsecos producidos a nivel vascular que causan su remodelación, la que se acentúa de acuerdo al tiempo de exposición del individuo a la hipoxia; además, se incrementa la producción de factores de crecimiento y del fibroblasto adventicial, alterando el tono vascular (Knot y Nelson, 1998). Animales sometidos a hipoxia se caracterizan por un incremento del tono vascular pulmonar que es mantenido, en parte, por una remodelación vascular dependiente de la duración y severidad de la misma; así, en la pared arterial pulmonar, la célula endotelial, el músculo liso y el fibroblasto adventicial tienen un crecimiento específico, que se puede observar aún a las 24 horas de exposición a la hipoxia. Las células arteriales producen factores de contracción o relajación en respuesta a varios estímulos, los cuales modulan el tono vascular (Brij Seema y Peacock, 1998).
Investigaciones recientes demuestran la existencia de sustancias vasoactivas que actúan en respuesta a hipoxia, tales como la Sustancia P (SP), un neuromodulador del grupo de neuropéptidos (NPS). Esta sustancia es un potente vasodilatador secretada por los nervios peri-vasculares y liberada dentro de las células endoteliales (Schaffer et al., 1998).

El presente trabajo tuvo como objetivo estudiar el rol del neuromodulador SP en el mecanismo íntimo de la HAP, utilizando el ave como un modelo experimental. Se pretende que los resultados del presente trabajo permitan orientar los estudios de adaptación a la altura en otras especies de producción.

\section{Mateliriales y Métodos}

El trabajo se realizó en los laboratorios de Fisiología Animal de la Facultad de Medicina Veterinaria de la UNMSM y de la Estación Experimental del C.I. IVITA-El Mantaro. Se utilizaron 30 pollos machos de producción de carne de la línea Arbor Acress.

Los pollos nacieron y fueron criados a nivel del mar hasta los 35 días de edad, luego de los cuales fueron expuestos durante tres días a 3,320 $\mathrm{m}$ de altitud.

En cada animal se determinó la Presión Arterial Pulmonar media (PAPm) mediante la técnica descrita por Burton et al. (1968), antes (Pre-SP) y después (Post-SP) de la aplicación de $0.30 \mathrm{mcg} / \mathrm{kg}$ de peso vivo de Sustancia P (SP) por vía directa en la arteria pulmonar. Para tal efecto se utilizó un transductor de presión modelo 167AC co- 
nectado a un fisiógrafo de registro térmico modelo 8805B, ambos de la marca Hewlett Packard.

Los resultados de la PAPm en cada estadío de Post-SP fueron expresados en valores promedio y comparados con el de PreSP, utilizando la prueba de " $\mathrm{t}$ " Student, teniendo en consideración las diferencias a través del tiempo. Se estableció el nivel de confiabilidad de la SP a través de la prueba de intervalo de confianza mayor y menor entre los promedios y las diferencias.

\section{Resultados y Discusión}

La PAPm antes y después de la administración del neuromodulador SP, muestra diversas tendencias de efecto (Cuadro 1), llegando a niveles por debajo de los registrados a nivel del mar (Burton et al., 1968).
La administración de SP disminuyó significativamente $(\mathrm{p}<0.001)$, la PAPm durante los 90 segundos de su administración alcanzando el promedio más bajo a los 45 segundos, para luego iniciar su incremento desde los 60 segundos hasta los 90 segundos, tendiendo a alcanzar el valor Pre-SP. El registro de $37.92 \pm 4.10 \mathrm{~mm} \mathrm{Hg}$, antes de la administración de SP, guarda estrecha relación con investigaciones realizadas en aves expuestas a similares altitudes (Burton et al., 1968; Cueva et al., 1974).

La Fig. 1 muestra gráficamente las diferencias de la PAPm en los diferentes intervalos de estudio después de la administración de la sustancia P con relación a la PAPm antes de la administración de la Sustancia P.

Al parecer, esta disfunción fisiológica en respuesta a la hipoxia, puede ser un mecanismo de defensa por el cual la vasculatura

Cuadro 1. Presión arterial pulmonar media (PAP) antes y después de la administración de Sustancia $\mathrm{P}$ en pollos de carne sometidos a hipoxia de altura $(3,320 \mathrm{msnm})$ durante tres días

\begin{tabular}{cc}
\hline $\begin{array}{c}\text { Tiempo de administración de } \\
\text { Sustancia P (seg) }\end{array}$ & $\begin{array}{c}\text { PAPm }(\mathrm{mmHg}) \\
\mathrm{x} \pm \text { d.e. }^{2}\end{array}$ \\
\hline 0 & $37.92 \pm 4.10$ \\
1 & $36.08 \pm 3.70^{*}$ \\
5 & $31.11 ? 7.51^{*}$ \\
10 & $28.00 ? 10.22^{*}$ \\
15 & $26.43 \pm 11.02^{*}$ \\
30 & $24.43 ? 11.02^{*}$ \\
45 & $23.17 \pm 11.54^{*}$ \\
60 & $24.08 \pm 10.98^{*}$ \\
75 & $27.27 \pm 10.97^{*}$ \\
\hline 0 & $30.67 \pm 9.40^{*}$ \\
\hline
\end{tabular}

${ }^{1}$ Solución hídrica al $0.01 \%$ de SP en dosis $0.30 \mathrm{mcg}(0.3 \mathrm{ml}) / \mathrm{kg}$ de p.v.

2 Promedio \pm desviación estándar

* Diferencia significativa $(\mathrm{p}<0.001)$ con relación al estadío Pre-SP 


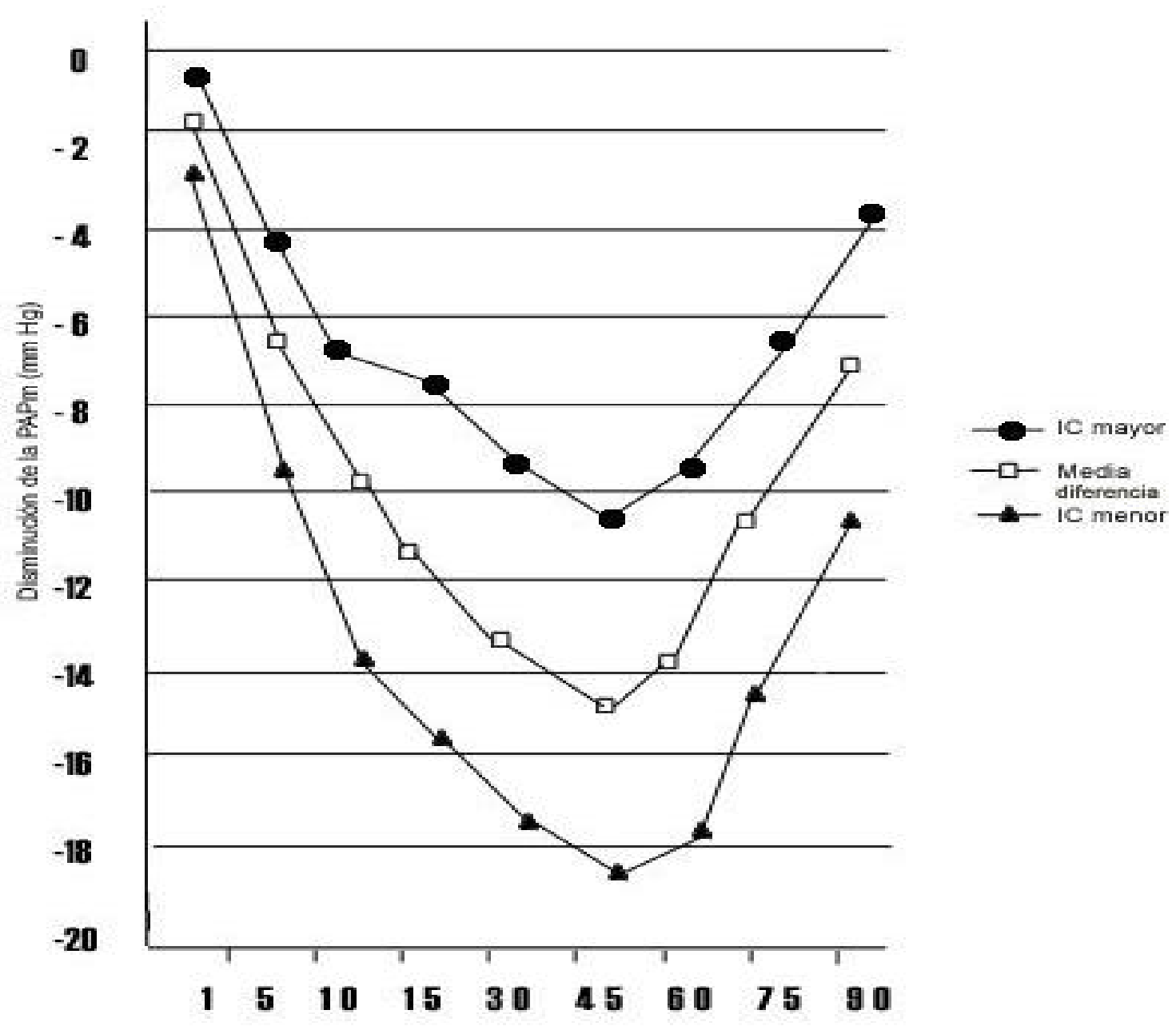

Tiempo (S)

Figura 1. Representación gráfica del efecto de la SP sobre la PAPm. Obsérvese los valores e intervalos de confianza a diferentes tiempos Post-SP en relación al valor Pre-SP $(\mathrm{mmHg})$

se protege ante cualquier tipo de injuria del área del lecho vascular a fin de mantener un menor flujo en esas zonas donde tienen incrementadas su permeabilidad, por lo tanto incrementado su presión capilar (Zanaboni et al., 1992; Staton e Ingram, 1998, Kato et al., 1990). La presión intravascular causa un potencial de despolarización de la membrana abriendo canales de $\mathrm{Ca}^{++}$dependiente de voltaje y actuando como un censor de voltaje. De esta manera, se incrementa la entrada de $\mathrm{Ca}^{++}$a la pared arterial produciendo la despo- larización del músculo liso (células musculares lisas), y la vasoconstricción arterial vista en pequeñas arterias; además, suele ocurrir una gran constricción entre cada tono miogénico (Meininger y Davis, 1992; Knot y Nelson, 1998). A una presión dada, el diámetro arterial es muy sensible al potencial de membrana, llegando a la hiperpolarización de la misma, que a su vez origina vasodilatación; un mecanismo común para muchos vasodilatadores sintéticos y endógenos con activación de canales de $\mathrm{K}^{+}$. Además, el proceso 


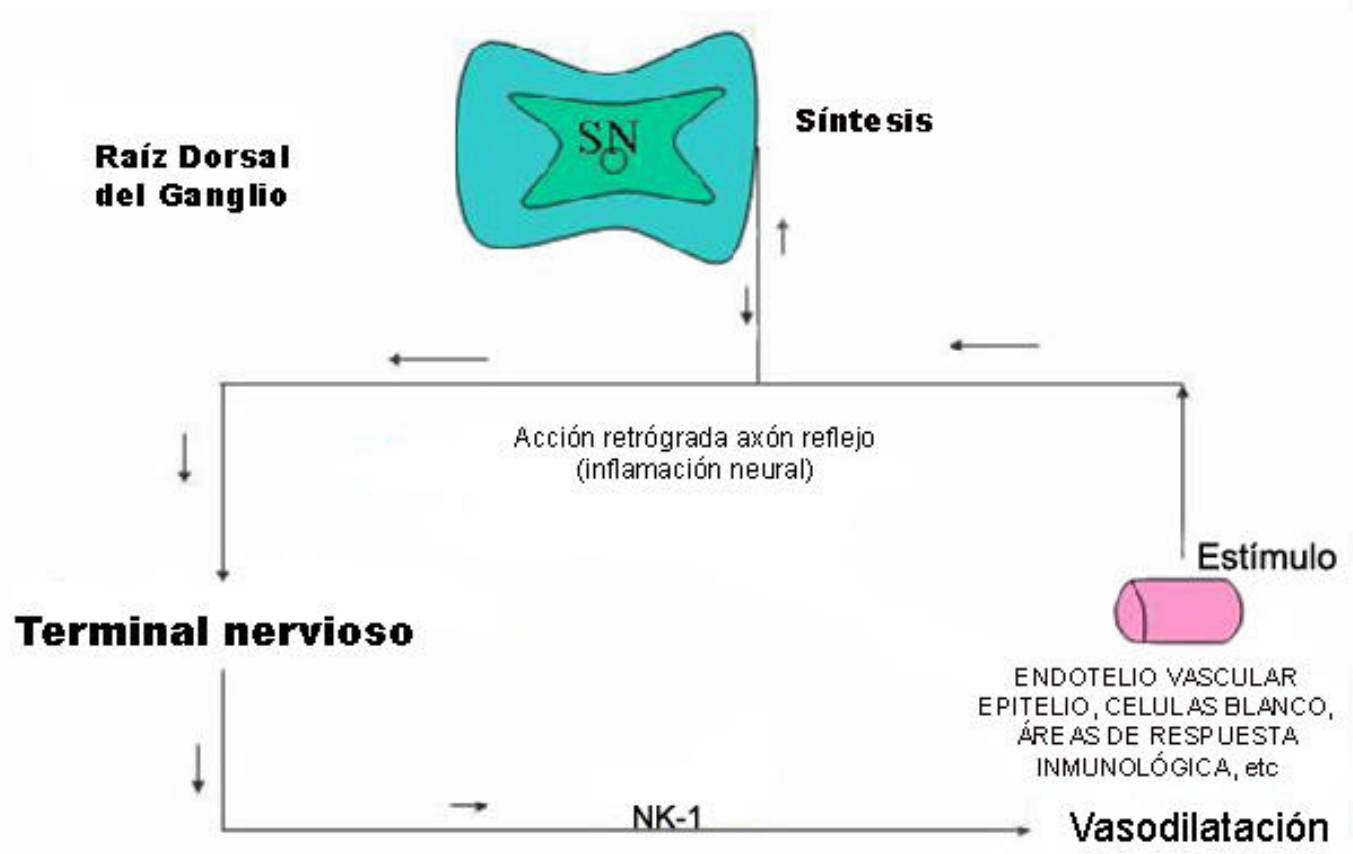

Figura 2. Posible mecanismo de acción de la SP

de generación y mantenimiento del ritmo respiratorio está controlado por la excitabilidad de las neuronas y la conductancia al $\mathrm{K}^{+}$. Dos canales de $\mathrm{K}^{+}$están esencialmente involucrados en la respuesta vasomotora: canales de $\mathrm{K}^{+}$sensibles al ATP que responde a cambios metabólicos celulares y canales de $\mathrm{K}^{+}$ estimulados por niveles de $\mathrm{Ca}^{++}$citosólico (Perrault y De Marte, 1993). Estos canales de $\mathrm{Ca}^{++}$en la hipoxia contribuyen a la respuesta del centro respiratorio (Mironov y Rickter, 1998; Sun y Reis, 1994).

La disminución de la PAPm en el presente estudio podría ser una respuesta al estímulo mecánico ejercido por el incremento del tono vascular o por un mecanismo que podría involucrar la hiperpolarización de la membrana, a partir de una vasodilatación dependiente del endotelio vascular (Perreault y De Marte, 1993; Armstead, 1996). Además, este factor de hiperpolarización dependiente del endotelio (EDHF) induce hiperpolarización del músculo liso vascular por activación de la bomba $\mathrm{Na}-\mathrm{K}$ ATPasa o bomba de $\mathrm{Na}$ (Brayden 1990; Feletou y Van Houtte, 1988) o por incremento de la conductancia de la membrana al K+ (Komori y Suzuki, 1997; Chen et al., 1988; Smith et al., 1991).

El posible mecanismo de acción de la Sustancia P se presenta en la Fig. 2. Considerando que numerosos neuropéptidos están localizados en terminales nerviosos mielinizados (AC) y amielinizados (C), y la estimulación antidrómica de estas fibras induce liberación de neuropéptidos almacenados, esto resulta en vasodilatación e incremento de la permeabilidad vascular por inflamación neurogénica. Estos impulsos nerviosos viajan no sólo centralmente, sino que a través de las ramificaciones colaterales pasan antidrómicamente para estimular los terminales nerviosos y causar liberación de neuropéptidos denominado "Axón reflejo". Tales agentes pueden actuar normalmente sobre receptores de terminales nerviosos pre y post sinápticos localizados sobre la célula blanco (Holzer, 1988; Hokfelt et al., 1994). Este mecanismo de acción podría estar ocurriendo con la SP, para producir el efecto vasoactivo sobre la arteria pulmonar (célula blanco), y por consiguiente disminuir la PAPm. 


\section{CONCLUSIONES}

- La administración de Sustancia P al $0.01 \%$ en dosis de $0.30 \mathrm{mcg}(0.3 \mathrm{ml}) / \mathrm{kg}$ de peso vivo en pollos de producción de carne expuestos durante tres días a hipoxia ambiental, disminuyó la PAPm en forma rápida.

- La disminución rápida de la PAPm permite sugerir la influencia de la Sustancia $\mathrm{P}$ a nivel endotelial vascular, modulando el efecto presor mediante la hiperpolarización de la membrana.

- El efecto hipotensor fue transitorio, ya que desde los 60 hasta 90 segundos PostSP se observó un incremento tendiente a acercarse al valor Pre-SP.

\section{Literatura Citada}

1. Alexander, A.F.; D.F. Will; R.F. Grover; J.T. Reeves. 1960. Pulmonary hypertension and right ventricular hypertrophy in cattle at high altitude. Amer. J. Vet. Res. 21: 199-204.

2. Armstead, W.M. 1996. Role of ATPsensitive $\mathrm{K}^{+}$channels in c GMP-mediated pial artery vasodilation. Am. J. Physiol. 270: H423-426.

3. Brayden, J.E. 1990. Membrane hyperpolarization is a mechanism of endothelium-dependent cerebral vasodilation. Am. J. Physiol. 259: H668-H673.

4. Brij Seema, O; A.J. Peacock. 1998. Cellular responses to hypoxia in the pulmonary circulation. Thorax 53: 10751079.

5. Burton, R.R; E.L. Besch; A.H. Smith.1968. Effect of hypoxia on the pulmonary arterial blood pressure of the chicken. Am. J. Physiol. 214: 1438-1442.

6. Cueva, S.; H. Sillau; A. Valenzuela; H. Ploog. 1974. High altitude pulmonary hypertension and right heart failure in broiler chicken. Res. Vet. Sci. 16: 370374.
7. Chen, G.; H. Suzuki; A.H. Weston. 1988. Acetylcholine releases endothelium-derived hyperpolarizing factor and EDRF from rat blood vessels. J. Pharmacol. 95: 1165-1174.

8. Feletou, M.; P.M. Van Houtte. 1988. Endothelium-dependent hyperpolarization of canine coronary smooth muscle. $\mathrm{Br}$. J. Pharmacol. 93: 515-524.

9. Grover, R.F. 1966. Comparative physiology of hypoxia pulmonary hypertension. En: Proc. Int. Symp. Card. Vasc. Resp. Effects hypoxia. Karger. Basel. New York. p 307-321.

10.Hokfelt, T.; H.G. Shaible; R.F. Schmidt. 1994. Neuropeptides nociception and pain. Chapman \& Hall. Weinheim, Germany.

11. Holzer, P. 1988. Local effectors functions of capsaicin sensitive sensory nerve ending: Involvement of tachykinins, calcitonin generelated peptide and other neuropeptide. Neuroscience 24: 739-768.

12. Kato, T.; Y. Iwama; K. Okumura; $\mathrm{H}$. Hashimoto; T. Ito; T. Satake. 1990. Prostaglandin $\mathrm{H} 2$ may be the endothelium-derived contracting factor released by acetylcholine in the aorta of the rat. Hypertension 15: 475-481.

13. Knot, H.J.; M.T. Nelson. 1998. Regulation of arterial diameter and wall $\mathrm{Ca}^{2+}$ in cerebral arteries of rat by membrane potential and intravascular pressure. J. Physiol. 508: 199-209.

14. Komori, K.; H. Suzuki. 1987. Electrical responses of smooth muscle cells during cholinergic vasodilation in the rabbit saphenous artery. Cir. Res. 61: 586-593.

15. Meininger, G.A.; M.J. Davis.1992. Cellular mechanisms involved in the vascular myogenic response. Am. J. Physiol. 263: H647-H659.

16. Mironov, S.L.; D.W. Rickter. 1998. Ltipe $\mathrm{Ca}^{2+}$ channels in inspiratory neurones of mice and their modulation by hypoxia. J. Physiol. 512: 75-87.

17. Perreault, T.; J. De Marte. 1993. Naturational changes in endotheliumderived relaxations in newborn piglet 
pulmonary circulation. Am. J. Physiol. Heart Cir. Physiol. 264: H302-309.

18. Schaffer, M.; T. Beiter; H.D. Becker; T.K. Hunt. 1998. Neuropeptides: Mediators of inflammation and tissue repair?. Arch. Surg. 133: 1107-1116.

19. Smith, J.C.; H.H. Ellenberger; K. Bellanyi; D.W. Richter; J.L. Feldman. 1991. Pre-Botzinger complex: A brain stem regions that may generate respiratory rhythm in mammals. Science 254: 726-729.

20.Sun, M.K.; D.J. Reis. 1994. Hypoxiaactivated $\mathrm{Ca}^{++}$currents in pacemaker- neurones of rat rostral ventrolateral medulla in vitro. J. Physiol. 476: 101-106.

21. Staton, G.; R. Ingram. 1998. Respiratory pulmonary edema. Scientific American INC. 1-8.

22. Will, J.A; E.G. Bisgard. 1975. Comparative homodynamic of domestic animals at high altitude. Prog. Res. 9: 138-43.

23. Zanaboni, P.B.; J.D. Bradley; R.O. Webster; T.E. Dahams. 1992. Cyclooxigenase inhibition prevents PMAInduced in pulmonary vascular permeability to album. J. APPL Physiol. 73: 2011-2015. 